Author Accepted Version. Final version published as: Temple, J. B., \& Dow, B.

(2018). The unmet support needs of carers of older Australians: prevalence and mental health. International psychogeriatrics, 30(12), 1849.

\title{
The Unmet Support Needs of Carers of Older Australians: \\ Prevalence and Mental Health
}

\author{
Jeromey Temple, $\mathrm{PhD}$ \\ Associate Professor \\ Demography and Ageing Unit \\ Melbourne School of Population and Global Health, \\ University of Melbourne \\ $\&$ \\ Briony Dow, PhD \\ Associate Professor \\ Demography and Ageing Unit \\ Melbourne School of Population and Global Health, \\ University of Melbourne \\ Director, National Ageing Research Institute NARI
}

Please direct correspondence to Jeromey B. Temple, Associate Professor, Melbourne School of Population and Global Health, 207 Bouverie St, University of Melbourne, Melbourne, VIC, 3010 Australia (Jeromey.Temple@unimelb.edu.au). Ph: +61 3 90359900 


\begin{abstract}
Background: Population ageing places greater demands on the supply of informal carers. The aims of this study were to examine (1.) the types of unmet support needs of carers of older Australians, and (2.) the association of unmet needs with mental health.
\end{abstract}

Methods: Utilising new data from the 2015 ABS Survey of Disability, Ageing and Carers, we calculated the prevalence of carers experiencing specific and multiple unmet needs for support, using single and multiple item measures. Logistic regression models were fitted to examine the association between unmet needs and psychological distress (using the Kessler psychological distress scale), once demographic and health factors were controlled for.

Results: In 2015, 35\% of carers of older Australians cited at least one unmet need for support. Among this group, almost two thirds cited multiple unmet support needs (64.7\%). The most prevalent types of unmet needs included financial (18\%), physical $(13 \%)$ and emotional support (12\%), as well as additional respite care and support to improve carer health (12\%). After controlling for demographic and health characteristics of the carer, having any unmet need for support increased the odds of psychological distress two fold $(\mathrm{OR}=2.20,95 \% \mathrm{CI}=1.65,2.94)$. With each successive unmet need for support, the odds of psychological distress increased 1.37 times $(\mathrm{OR}=1.36,95 \% \mathrm{CI}=1.22,1.54)$. Those who had received assistance with care, but required further support were 1.95 times more likely $(\mathrm{OR}=1.95,95 \% \mathrm{CI}=1.17,3.24)$ to be in distress and those who had not received care assistance were about 2.4 times more likely $(\mathrm{OR}=2.3895 \% \mathrm{OR}=1.56,3.62)$ to be in distress relative to those with no unmet need. 
Conclusions: Addressing unmet support needs of carers is important, not only for the planning of services for carers in an ageing population, but also because of the association between unmet support needs and carers mental health.

Keywords: Caring, Informal Care, Ageing, Psychological Distress 


\section{The Unmet Support Needs of Carers of Older Australians: Prevalence and Mental Health}

\section{Introduction}

With the speed of population ageing hastening across most high-income countries, the care needs of the growing number of older citizens has been an important priority for policy makers. Consistent with the preferences of older persons, successive Australian governments have sought to assist older people to receive care in their own homes, rather than institutions since the 1980s (Dow et al, 2013). One of the factors key to the success of this strategy is the large pool of informal carers in Australia, providing unpaid caring activities, estimated to account for $80 \%$ of care of older people in Australia (Productivity Commission, 2011). In 2015, 2.7 million people providing informal care with an estimated economic value of $\$ 60$ billion (ABS, 2016; Deloitte Access Economics, 2015). The UK and USA also rely significantly on informal carers to care for older persons. Six and a half million people in the UK are carers, with $44 \%$ caring for a parent or grandparent (Carers UK, 2015) and 34.2 million Americans provide unpaid care to a person aged over 50 (National Alliance for Caregiving, 2015).

Although informal caring is mostly unpaid, a number of government programs and payments are in place to assist Australian carers. Carers can access respite care and several types of carer payment, including an income support payment for those who are unable to work in paid employment due to their caring role through the government's Carer Gateway (Australian Government, 2017). There are carer advocacy and support services in every state and territory through which carers can gain access to services such as counselling and support groups (Carers Australia, 2017). Carers in Australia 
also have the right to request flexible working arrangements enshrined in legislation and this request cannot be reasonably refused by employers (Victorian Equal Opportunity and Human Rights Commission, 2012). There are similar arrangements in place for carers in the UK but in the USA there is no income support payment for carers (Agingcare, 2017).

Despite these government programs, research has shown that many carers have unmet needs for further support. Some carers require support and education on practical skills to assist with their caregiving role and access to support services is oftentimes limited due to complex eligibility criteria (Essue et al., 2010). This is complicated further by the low levels of assistance to primary caregivers and awareness of many existing community services for carers and their recipients (Lucke et al., 2008; Vecchio, 2008b). Users of different support services may also have differing levels of unmet support needs. In an examination of respite service use in 2003, respite care users had considerable unmet demands for financial assistance, whereas non respite care users had unmet needs to improve their own well-being (Vecchio, 2008). More generally, stressors associated with care giving may impact upon the carer, requiring additional needs, including health and emotional support (Horner and Boldy, 2008; O'Connell et al.,2003). This issue of unmet emotional support is important, as there is considerable evidence that carers are at increased risk of adverse mental health, including depression (Dahlberg et al, 2007, Neri et al, 2012, Cummins et al, 2007, Gaugler et al, 2004).

Despite the considerable contribution of the above studies to our understanding of carer needs, there remains a gap in studies of the types of needs of carers: specifically among those who care for older Australians and those who have multiple unmet support needs. 
With the availability of new nationally representative data, we seek to answer two questions: (1) what is the prevalence and type of unmet support needs of those who care for older Australians? and (2) is there an association between the carers unmet support needs and mental health?

\section{Methods}

Data

Data for this study are from the 2015 Survey of Disability, Ageing and Carers (SDAC) conducted between July and December 2015. Three populations were sampled using multi-stage sampling techniques. These consisted of persons living in private dwellings, in self-care retirement villages and in cared accommodation. The module on carers unmet needs was administered to primary carers living in households. Primary carers were identified through a series of screening questions. As defined by the ABS, primary carers "provide(s) the most informal assistance, in terms of help or supervision, to a person with one or more disabilities, with one or more of the core activities of mobility, self-care or communication" (ABS, 2016). The ABS has the authority to collect these data under the Census and Statistics Act 1905, and has collected 8 cross sections since 1981. Respondents were not paid for participation nor were any other inducements offered.

Of 31,957 households originally contacted, 25,555 fully responded, yielding a response rate of $80 \%$. Of these persons living in households, 2,421 were primary carers who filled out the unmet needs questionnaire. Of this group, we omit 340 cases where the primary carer did not answer questions pertaining to unmet needs. This left a final sample size of 2081 primary carers. Within this group approximately $18 \%$ care for 
children $(n=382), 20 \%$ care for person in the primary working ages $(n=420)$ and $61 \%$ care for older Australians aged 55 and over $(n=1,274)$. This later group of carers are the focus of this study, representing over $60 \%$ of primary carers. We used the cut-off age of 55 because from age 55, persons born before 1960 can access private superannuation and retire in Australia. The average age of primary carers (of recipients of any age) in Australia is 55, with two thirds being female and the majority caring for a family member (ABS, 2016b).

\section{Measures}

In the self-completed carer questionnaire, primary carers were asked "Do you feel you need more support or an improvement in your situation to aid in your role as a carer?" A follow up question was then asked "Which of the following would assist you in your role as a carer?" A list of possible responses was than provided, including:

- More respite care

- More financial assistance

- More physical assistance

- More emotional support

- An improvement in my own health

- More aids/equipment to help me assist in my role as a carer

- More courses available on how to care for persons with particular disabilities

- More training in correct use of equipment

- More training in correct methods of lifting to prevent injury to myself

- None of the above 
For those who answered multiple needs, they were asked to nominate which would "most assist you in your role as carer?" Herein, this later measure is referred to as the single item measure.

For the first time in 2015, the SDAC collected measures of psychological distress. The specific measure of psychological distress used was Kessler's K10. The K10, although not a diagnostic tool, is a widely used screening instrument for psychological distress based on the respondent's emotional state in the 4 weeks prior to the interview (ABS, 2016; Anderson et al., 2013). This enables an analysis of whether psychological distress is associated with unmet carer support needs. Following other examples in the literature, we indicate a 'high' or 'very high' score on Kessler's measure as indicating psychological distress (Anderson et al, 2013).

\section{Statistical Model and Estimation of Variance}

To examine differences in the prevalence of unmet carer needs and psychological distress we present weighted tests of proportions, with a 95\% critical value. To examine the association between unmet carer needs and psychological distress we fitted logistic regression models. Using the raw logit coefficients, we calculated odds ratios (OR) which measure the change in the odds of experiencing psychological distress given a change in unmet carer needs, once all other factors in the model are controlled for.

Variables were entered into the regression and improvement to model fit assessed using the Bayesian Information Criteria following Raftery's procedure (Raftery, 1995). With all models specified, we checked the conditioning of the matrix of independent variables to investigate any collinearity influence (Belsley et al, 1980). The condition 
numbers and variance inflation factors were small providing support for the model specification. Final goodness-of-fit for the logistic regression models was confirmed using the Hosmer and Lemeshow test (Hosmer et al, 1997).

Due to the complex survey design, adjustments are necessary to generate correct variance estimates. The SDAC includes 60 replicate weights on the data file to adjust for sample design and non-response. Utilizing an algorithm developed by Winter (2008), we employed the unstratified delete-one jackknife method to make the necessary replicate adjustments (Wolfer, 1985). All analyses were conducted using Stata 15 (Stata Corporation, 2017).

\section{Results}

Table 1 displays the differences in prevalence of carer unmet support needs using the single item ("most assist you") and multiple item measure ("any need for assistance"). Regardless of the measure used, about one third of the primary carers of older persons have unmet demands for support (34.6\%). There is considerable variation in the prevalence of additional support requested by carers according to the measure used (Table 1). Relying only on the single item measure significantly underrepresents the full demand for a number of sources of support. For example, using the single item measure, about $9 \%$ of carers report 'more financial assistance' compared with almost 1 in 5 carers responding to the multiple item measure (17.7\%). Similarly, only 3\% report a need for emotional support on the single item measure, but about $12 \%$ of carers indicate this unmet need on the multiple item measure $(11.9 \%)$. 


\section{[TABLE 1]}

Focusing on the multiple item measure, financial assistance is the most heavily cited among carers as an unmet need (17.7\%). Half of all carers with unmet needs cited this unmet support need (49.7\%). In addition, over 10\% of carers report additional physical assistance (12.7\%), support with improving their own health (12.1\%), more emotional support (11.9\%) and respite care (11.6\%) as key support needs to improve their caring role. Around $6 \%$ of carers report a need for additional aids or equipment $(6.3 \%)$ or courses on caring for persons with particular disabilities $(5.8 \%)$.

Using the multiple item measure, we generate a variable measuring the number of types of unmet needs of carers. In Table 2, we tabulate the types of unmet needs by number of types of unmet needs. This table includes the population of those who report any unmet need $(n=445)$. Of those with unmet needs, $35 \%$ of carers have one unmet need only, $43 \%$ have 2 or 3 unmet needs and about 1 in 5 have 4 or more unmet needs $(21.5 \%)$. In total, almost two thirds of carers with unmet needs have multiple unmet needs (64.7\%), representing 22\% of all primary carers of older Australians.

\section{[TABLE 2]}

As expected, the proportions reporting across all unmet support need types increase with total unmet needs. For example, $7 \%$ of those with one unmet need require physical assistance, compared with half of those with 2 or 3 unmet needs (47.7\%) and almost two thirds of those with 4 or more unmet needs (63.9\%). Of those with multiple needs, considerable proportions of these groups cite financial, physical and emotional support. 
However, those with 4 or more needs also have considerably high demand for training and courses compared with those who have fewer unmet needs. Half of those with four or more unmet needs have unmet demand for "more aids/equipment" $(52.1 \%)$ or "courses available on how to care for persons with particular disabilities" compared with under $5 \%$ of those with 1 unmet need and under $15 \%$ of those with 2 or 3 unmet needs.

The results above indicate a considerable proportion of carers report unmet and multiple unmet support needs, and that those with higher numbers of needs report demand for support across a range of need types. An important question, therefore, is can unmet needs place additional stressors upon carers influencing their mental health? As these data are cross sectional, we cannot infer causation, but we can investigate whether there are differences and an association between increasing likelihood of unmet needs with increased likelihood of psychological distress.

Before turning to the multivariate analyses, results in table 3 illustrates the differences in demographic characteristics and care details of carers tabulated by whether they have unmet support needs. Consistent with evidence on carers of persons of any age reported by the ABS, about two thirds of carers of older Australians are female (64\%) (ABS, 2016b). About $70 \%$ of this group were born in Australia (72\%), are married (69.7\%), living in a major city $(67.1 \%)$ co-resident with the recipient of care $(70.3 \%)$ and aged between 45 and $74(71.6 \%)$.

\section{[TABLE 3]}


Comparing across unmet support need status, those with unmet needs tend to be slightly older, married and not in the labour force and with lower levels of education compared to those with no unmet support needs. It is not surprising then that about $52 \%$ of those with unmet support needs are caring for a spouse, compared with $40 \%$ of those with no unmet needs. Those with no unmet support needs are also slightly more likely (48.6\%) to have used support services compared to those with no unmet needs $(40.6 \%)$. There are no meaningful differences between the groups by duration of care or the carers number of long term conditions.

Results in Table 4 illustrate the differences in the prevalence of psychological distress in this group of careers of older Australians (60\% of all primary carers) by the demographic and care characteristics provided above. Among this group of carers, approximately one third of those with any unmet support needs are in psychological distress $(31.3 \%)$, compared with $17 \%$ of those with no unmet needs. We also observe considerable differences in the prevalence of psychological distress by the number of unmet support needs. Compared to the $17 \%$ of those with no unmet needs in distress, about $26 \%$ of those with 2 or more needs and over half of those with 3 or more needs $(52.6 \%)$ report psychological distress.

\section{[TABLE 4]}

To examine the association between unmet needs and psychological distress, it is important to control for demographic groups with differing prevalence rates. For example, variations in psychological distress in these data appear by age, gender, 
country of birth, education level and the number of health conditions that the carer reports (Table 4).

With extensive demographic controls, the multivariate evidence strongly support the proposition that unmet support needs of carers is associated with carers' mental health (Table 5). With control variables included, having any unmet need for support increases the odds of psychological distress two fold (Model $1 \mathrm{OR}=2.20 \mathrm{p}<0.001$ ). Including a continuous measure of unmet support needs, we find that with each additional unmet support need, the odds of carer psychological distress increases 1.37 times (Model 2 $\mathrm{OR}=1.36 \mathrm{p}<0.001)$. Further support is provided in Model 3 which includes a categorical variable measuring combinations of receipt of whether the carer received any assistance to care and unmet need. Not surprisingly, there is no difference in distress between those who do not have unmet support needs classified by care assistance usage $(\mathrm{OR}=$ $0.98 \mathrm{p}<0.10$ ). However, those who have received care assistance and have continuing unmet needs are about 1.95 times more likely to be in distress relative to those with no unmet needs $(\mathrm{OR}=1.95,95 \% \mathrm{CI}=1.17,3.24)$. Those who have unmet needs and have not received care assistance are at almost 2.4 times the risk of distress (OR $=2.3895 \%$ $\mathrm{CI}=1.56,3.62)$

\section{[TABLE 5]}

Previous studies highlight the impact of dementia on carers levels of stress and wellbeing (Bertrand et al, 2006; Stirling et al, 2010; Gaugler et al, 2004). In the confidentialised data we utilise, the care recipient's conditions are only available for co-resident carers. That is, for primary carers living in households who have non usual 
resident care recipients, the condition data is unfortunately unavailable. Restricting our sample to 890 co-resident carers, we re-estimated all models measuring a care recipient with dementia (Model 4). Unsurprisingly, carers with a care recipient who has dementia are at an almost 2 fold risk of distress compared to those caring for a person without dementia $(\mathrm{OR}=1.8995 \% \mathrm{CI}=1.01,3.5)$. Importantly, even with the additional controls for dementia and the restricted sample size, the coefficients measuring unmet needs and receipt of care assistance are highly comparable to the full sample models. That it, those who have received care assistance and have unmet needs are significantly more likely to be in distress $(\mathrm{OR}=1.9195 \% \mathrm{CI}=1.02,3.55)$ as are those who have unmet needs but no care service use $(\mathrm{OR}=2.795 \% \mathrm{CI}=1.70,4.29)$.

Although not the purpose of the analyses, the control variables are also of substantive interest themselves. Among this group of carers of older Australians, psychological distress is highest among females (versus males), those from a non-English speaking background (versus Australian born), younger carers and those with low levels of education (versus university educated). The number of health conditions of the carer are also strongly associated with the odds of psychological distress $(\mathrm{OR}=1.39 \mathrm{p}<0.001)$. A range of other characteristics, included marital status, geography, income, carer relationship and co-residence status were tested and found not to be significant predictors of psychological distress in this sample. Polynomial terms for the number of health conditions and unmet needs, as well as various interaction terms were also not significant. Both models show absence of multicolinearity (Mean VIF $=1.46$ ) or omitted variable bias and the final specification is confirmed with the HosmerLemeshow test $(\mathrm{Chi}=9.4 \mathrm{p}<0.1$ model $1 ; \mathrm{Chi}=4.1 \mathrm{p}<0.1$ model 2$)$. All models further control for duration of care and prior use of care services. 


\section{Discussion}

Like most high-income nations such as the USA and UK, Australia's population is projected to age considerably out to and beyond 2050 (ABS, 2013). The Australian government, along with older persons themselves, prioritise care in the community, rather than institutional settings. This places considerable faith, but also pressure upon the informal care workforce in Australia, numbering in excess of 2.7 million people. Supporting the needs of carers is therefore crucial. In this study, we sought to (1.) examine what are the unmet care needs of the primary carers of older Australians and (2.) to investigate whether there is an association between these needs being unmet and the carers mental health.

\section{Types of Unmet Support Needs}

In 2015, 35\% of carers of older Australians cited at least one unmet need for support. Among this group, almost two thirds cited multiple unmet support needs (64.7\%). The most prevalent types of unmet needs included financial (18\%), physical (13\%) and emotional support (12\%), as well as additional respite care (12\%). These findings are consistent with earlier surveys of carers in Australia (Cummins et al, 2007; Schofield et al, 1998). The Cummins et al (2007) study found that, "satisfaction with ability to pay for household essentials, to afford the things you would like to have, to save money, to have financial security, and to not worry about income covering expenses, are all severely comprised for carers compared with a general population sample ( $p$ vii)." They also found that carers were much less likely to be in paid employment compared with 
the general population with $20.6 \%$ of their sample classifying themselves as unemployed (Cummins et al, 2007).

Our findings show that carers still have significant unmet financial needs. This could be related to the well-documented difficulties that carers have in combining paid work with caring, especially primary carers. It could also mean current carer payments are not adequate to meet the financial needs of those who rely on these payments. Future research could include a review of the effectiveness of current workplace arrangements that seek to enable carers to combine paid work with a caring role and/or a review of the adequacy of carer payments from government.

Other unmet needs we identify (physical and emotional support and additional respite care) are potentially due to lack of services and/or poor tailoring of these services for the diversity of carer needs. Despite carers often identifying respite care as a need, they rarely take up the current respite offerings, as they are either inconvenient or difficult to access for the carer or do not provide suitable activities or care for the care recipient (Phillipson, 2016). Respite services should be better tailored to meet carers' and carer recipients' specific needs, including offering culturally specific programs to carers from culturally and linguistically diverse background (Koloski et al, 2002).

It is interesting to note that those with multiple support needs were significantly more likely to signal needs for training and aids to assist in their caring role. There are existing free on-line training programs for carers of people with specific conditions usually associated with older age, such as dementia, in Australia. This study suggests that these could be better targeted to those carers who report a number of unmet needs. 
Results here also underscore the importance of measurement in the unmet needs of carers. Focusing only on a single item measure of the 'most assist you' significantly under represents the prevalence of a range of unmet needs of carers, which is important as two thirds of those with unmet needs have multiple unmet support needs. As such, usage of the single item measure significantly distorts the types of overall needs of carers with multiple unmet needs, particularly as it pertains to needs for training and aids to assist in their caring role. This may have implications for health and age care professionals working to support carers. Careful wording of questions aimed to assess carers' unmet needs is required. Rather than asking a single question of carers as to what they think would be of most assistance, our findings suggest that a checklist with a range of options would enable carers to better identify their unmet needs.

\section{Association between Unmet Needs and Mental Health}

This measurement issue is noteworthy, as results of this study point to an important association between increasing unmet needs of carers and a higher likelihood of psychological distress. Accounting for complex survey design and incorporating extensive control variables, we find that having any unmet need for support increases the odds of psychological distress two-fold and that each additional unmet need increased the likelihood of psychological distress by $36 \%$. Given that two thirds of carers in this sample have multiple unmet support needs, this result is concerning.

Carer depression has been found to be a risk factor for placement of the older care recipient in residential aged care (Gaugler et al, 2009) and abuse of the care recipient (Cooper et al, 2010) so it is a key risk factor for adverse outcomes in the care 
relationship. But it is also important for the health and wellbeing of the carer themselves, as increased depression is associated with reduced quality of life (Bruvika et al, 2012) and increased suicidal ideation in carers (O'Dwyer et al , 2013). Most of the research on caregiving has focused on characteristics of the carer, care recipient and the relationship between them rather than broader social and policy issues (Dow \& McDonald, 2003). While previous research has identified important directions for mental health clinicians (for example, Loi et al, 2016), this study points to the need to consider broader policy issues, such as income support, as many carers clearly have unmet financial needs that may impact their mental health.

The availability of government programs, however, does not necessarily mean that carers take them up and/or that they reduce burden or mental distress. In Australia, less than $30 \%$ of carers use respite services (AIHW, 2007). There has been considerable investigation of why carers do not use respite services and the evidence is mixed. For example, some studies show spousal carers are less likely to use respite than nonspousal carers (Robinson et al, 2005). Other studies have not found any relationship between carer relationship and use of respite services (Douglass \& Visconti 1998, Douglass \& Fox 1999, Montoro-Rodriguez et al. 2003). Indeed, some studies show carer or caregiver characteristics are only weakly associated with non-use of services (Phillipson et al, 2013). It may simply be that these services are difficult to access or do not meet carers' or care recipients' needs (Phillipson, 2016). For example, people from a culturally and linguistically diverse background are more likely to use services that are specifically tailored to their culture (Koloski et al, 2002). 
Apart from barriers and usage to respite care, there have been very few studies in Australia on barriers and applications for financial support, for example through the Carers Payment or Carers Allowance. This is a considerable research gap and further analysis is warranted here.

\section{Limitations and Extensions}

In interpreting these results, it is important to recognize the studies limitations. Firstly, the data are cross sectional. We cannot and do not draw causal inferences about carers unmet needs and psychological distress. Unfortunately, there is a dearth of nationally representative longitudinal data with measures of carers needs and psychological wellbeing in Australia, in which this linkage could be explored further.

An additional limitation of this study is that the SDAC measures focus only on primary carers. However, this group represents a substantial proportion of the total informal care workforce in Australia. Of the 2.7 million Australians providing informal care in 2015, approximately 856,000 , or about one third, were deemed to be primary carers by the ABS (ABS, 2016b). Our study represents a sub sample of about $60 \%$ of this group who specifically care for older Australians. By definition, however, primary carers provide the most informal care to the recipient, and therefore most likely to have unmet needs to assist in their care provision. However, these data do raise the question of how other carers' unmet needs for support are distributed throughout the population and whether the associations with mental health are as strong as we observe in this study? For example, do carers of younger care recipients have differing unmet needs and does this association with psychological distress persist to the same degree observed here? 
Analysis of longitudinal data to assess the relevant pathway from unmet support needs to psychological distress is a priority. Further studies may also wish to examine the relationship between unmet support needs and mental health in other countries with different funding and support mechanisms for carers and care recipients. Moreover, there is a need for further Australian studies on barriers to accessing means or alleviating financial stress - mainly through the Carers Allowance and Carers Payment. There is also considerable scope to examine the intersection of unmet needs, the care recipients health conditions and carers psychological wellbeing.

\section{Summary}

Notwithstanding these limitations, using newly released nationally representative data on Australian carers, we show the prevalence of unmet support needs to be considerable $(34.6 \%)$, that two thirds of this group have multiple unmet support needs and that unmet financial, emotional, physical and support to improve carers health are among the most commonly specified. Moreover, we show a clear association between unmet needs and psychological distress, independent of comprehensive control variables and accounting for complex survey design. We further show that even accounting for unmet needs, specific health conditions of the care recipient, such as dementia, heighten carers likelihood of psychological distress.

\section{Conflict of Interest}

None. 


\section{Description of authors' roles}

Author 1 and 2 jointly designed the study and authored the manuscript. Author 1 performed the data analysis.

\section{Acknowledgement}

Data for this study were made available to the authors by the Australian Bureau of Statistics (ABS). Author 1 is funded by the Australian Research Council's Centre of Excellence in Population Ageing Research (CE1101029). The opinions expressed herein are those of the authors alone.

\section{References}

ABS. (2016) Disability, ageing and carers, Australia: Summary of findings. Catalogue Number 4430.0, Australian Bureau of Statistics: Canberra.

ABS. (2016b) A profile of carers in Australia. Information Sheet, Australian Bureau of Statistics: Canberra.

ABS. (2013). Population Projections, Australia, 2012 to 2101. Catalogue Number 3222.0, Australian Bureau of Statistics: Canberra.

Agingcare (2017) https://www.agingcare.com/articles/quit-job-to-care-for-parents150227.htm, accessed August 2017. 
Australian Institute of Health and Welfare: Dementia in Australia: National data analysis and development. 2007 [http://

www.aihw.gov.au/publications/index.cfm/title/10368]. Canberra: AIHW

Anderson, T. M., Sunderland, M., Andrews, G., Titov, N., Dear, B. F. \& Sachdev, P.

S. (2013). The 10-Item Kessler Psychological Distress Scale (K10) as a Screening Instrument in Older Individuals. American Journal of Geriatric Psychiatry, 21, 596.

Australian Government. (2017). Carer Gateway. Available from: https://www.carergateway.gov.au/ Accessed August 2017.

Belsley D, Kuh E, Welsch R. (1980). Regression diagnostics: Identifying influential data and sources of collinearity. John Wiley \& Sons, Inc: New York.

Bruvika, F. K, Ulsteina, I D, Ranhoff, A H, Engedala, K. (2012). The Quality of Life of People with Dementia and Their Family Carers. Dementia and Geriatric Cognitive Disorders, 34, 7-14

Carers Australia (2017). http://www.carersaustralia.com.au/. Accessed August 2017.

Carers UK (2015). Facts about carers: policy briefing, Carers UK. London. file://C:/Users/bdow/Downloads/facts-about-carers-2015.pdf 
Cooper, C, Selwood, A, Blanchard M, Walker Z, Blizard R and Livingston G. (2010). The determinants of family carers' abusive behaviour to people with dementia: Results of the CARD study. Journal of Affective Disorders, 121, 136-142.

Cummins, R. A, Hughes, J, Tomyn, A, Gibson, A, Woerner, J, Luffana, L. (2007).

Special Report - The Wellbeing of Australians: Carer Health and Wellbeing. Australian Unity Wellbeing index, Survey 17.1, Report 17.1.

Dahlberg L, Demack S, Bambra C. (2007). Age and gender of informal carers: a population-based study in the UK. Health Soc Care Community, 15, 439-445.

Deloitte Access Economics (2015). The economic value of informal care in 2015. Report for Carers Australia, Access Economics: Canberra.

Dow, B and McDonald, J. (2003). Social Support or Structural Change? Social work theory and research on care giving. Australian Social Work, 56, 197-208.

Dow, B., Sparrow, P., Moore, K., Gaffy, E. and Yates, I. (2013). What do older Australians want? Australasian Journal on Ageing, 32, 236-240.

Essue, B. (2010). Informal care and the self-management partnership: implications for Australian health policy and practice. Australian Health Review, 34, 414-422.

Gaugler, J. E, Yu, F, Krichbaum, K, Wyman, J. F, (2009). Predictors of Nursing Home Admission for Persons with Dementia. Medical Care, 47, 191-198. 
Gaugler, J. E., Anderson, K. A., Leach, M. R., Smith, C. D., Schmitt, F. A., \& Mendiondo, M. (2004). The emotional ramifications of unmet need in dementia caregiving. American Journal Of Alzheimer's Disease And Other Dementias, 19(6), 369-380.

Horner, B and Boldy, D. (2008). The benefit of 'ageing-in-place' in an aged care community. Australian Health Review, 32, 356-365.

Hosmer D, Hosmer T, Le Cessie S, Lemeshow, S. (1997). A comparison of goodnessof-fit tests for the logistic regression model. Statistics in Medicine, 16, 965-80.

Kosloski K., Schaefer J., Allwardt D., Montgomery R. \& Karner T. (2002) The role of cultural factors on clients' attitudes toward caregiving, perceptions of service delivery, and service utilization. Home Health Care Services Quarterly 21 (3/4), 65-88.

Loi, S. M., Lautenschlager, N., Dow, B., Moore, K., Cyarto, E., Ames, D. \& Russell, M. (2016). Factors associated with depression in older carers. International Journal of Geriatric Psychiatry, 31, 294-301.

Lucke, J Russell, A Tooth, L Lee, C Watson, M Byrne, G Wilson, A Dobson, A. (2008). Few urban-rural differences in older carers' access to community services. Australian Health Review, 32, 684-690.

National Alliance for Caregiving (2015) Caregiving in the US, http://www.caregiving.org/wp- 
content/uploads/2015/05/2015_CaregivingintheUS_Executive-Summary-June4_WEB.pdf

Neri AL, Yassuda MS, Fortes-Burgos AC, Guariento, M. (2012). Relationships between gender, age, family conditions, physical and mental health, and social isolation of elderly caregivers. International Psychogeriatrics, 24, 472-483.

O’Connell, B. Bailey, S. Walker, A. (2003). Promoting the health and well being of older carers: A proactive strategy. Australian Health Review, 26, 78-86.

O’Dwyer, S, Moyle, W, Zimmer-Gembeck, M and De Leo D. (2013). Suicidal ideation in family carers of people with dementia: a pilot study. Geriatric Psychiatry $28,1182-1188$.

Phillipson, L, Jones, S C, \& Magee C. (2013) A review of the factors associated with the non-use of respite services by carers of people with dementia: implications for policy and practice Health and Social Care in the Community (2014) 22(1), 1-12

Phillipson, L (2016) Rethinking dementia care. Australian Journal of Dementia Care. http://journalofdementiacare.com/rethinking-respite-care/, accessed 24/11/17.

Productivity Commission. (2011) Caring for Older Australians, Report No. 53, Final Inquiry Report, Canberra. 
Raftery, A. (1995). Bayesian Model Selection in Social Research. Sociological Methodology, 25, 111-163.

Schofield, H., Bloch S., Herrman H., Murphy B. and Nankervis J (eds) (1998) Family Caregivers: Disability, illness and ageing. Allen and Unwin. Melbourne.

Stata Corporation. (2017) Stata/SE 15.0 for Windows 64 bit. College Station: Texas.

Stirling, C., Andrews, S., Croft, T., Vickers, J., Turner, P., \& Robinson, A. (2010).

Measuring dementia carers' unmet need for services - an exploratory mixed method study.BMC Health Services Research,10, 122. http://doi.org/10.1186/1472-6963-10$\underline{122}$

Vecchio, N. (2008). Understanding the use of respite services among informal carers. Australian Health Review, 32, 459-467.

Vecchio, N. (2008). The use of support systems by informal caregivers: An Australian experience. Australian Journal of Primary Health, 14, 27-34.

Victorian Equal Opportunity and Human Rights Commission. (2012). Mature-age workers and the Equal Opportunity Act: know your rights. Melbourne: Victorian Equal Opportunity and Human Rights Commission. 
Winter, N. (2008). SVR: Stata module to compute estimates with survey replication based standard errors. Available from:

https://ideas.repec.org/c/boc/bocode/s427502.html Accessed: March 2017.

Wolfer, K. (1985). Introduction to Variance Estimation. New York: Springer-Verlag. 


\section{University Library}

\section{- M M N E R VA A gateway to Melbourne's research publications}

Minerva Access is the Institutional Repository of The University of Melbourne

Author/s:

Temple, JB;Dow, B

Title:

The unmet support needs of carers of older Australians: prevalence and mental health

Date:

2018-12-01

Citation:

Temple, J. B. \& Dow, B. (2018). The unmet support needs of carers of older Australians: prevalence and mental health. INTERNATIONAL PSYCHOGERIATRICS, 30 (12), pp.1849-1860. https://doi.org/10.1017/S104161021800042X.

Persistent Link:

http://hdl.handle.net/11343/254623 